\title{
Anxiety and Suicidality in a Hospitalized Patient with COVID-19 Infection
}

\author{
Danny Epstein ${ }^{1}$, Wisam Andrawis ${ }^{1}$, Ari M Lipsky², Hiba Abu Ziad ${ }^{3}$, Moshe Matan $^{4}$ \\ ${ }^{1}$ Department of Internal Medicine 'B', Rambam Health Care Campus, Haifa, Israel \\ ${ }^{2}$ Department of Emergency Medicine, Rambam Health Care Campus, Haifa, Israel \\ ${ }^{3}$ Infectious Diseases Unit, Baruch Padeh Medical Center, Poria, Israel \\ ${ }^{4}$ Intensive Care Unit, Baruch Padeh Medical Center, Poria, Israel
}

Received: 08/04/2020

Accepted: 08/04/2020

Published: 09/04/2020

\begin{abstract}
How to cite this article: Epstein D, Andrawis W, Lipsky AM, Ziad HA, Matan M. Anxiety and suicidality in hospitalized patient with COVID-19 infection. EJCRIM 2020;7: doi:10.12890/2020_001651.
\end{abstract}

Conflicts of Interests: The Authors declare that there are no competing interests.

This article is licensed under a Commons Attribution Non-Commercial 4.0 License

\section{ABSTRACT}

We describe the case of a young man admitted due to mild COVID-19 infection. During his hospitalization in an isolation ward, he had no respiratory distress or fever but developed symptoms consistent with anxiety and insomnia. Despite the appropriate supportive intervention, on hospital day 7 , he attempted suicide by jumping from the third-floor ward. The patient underwent urgent surgery and was transferred to a level I trauma center under strict isolation. Our findings emphasize the importance of the mental health aspects of patients treated during the COVID-19 pandemic.

\section{LEARNING POINTS}

- The COVID-19 pandemic requires social distancing, quarantine and isolation, which may precipitate new psychiatric symptoms in people without mental illness or aggravate existing conditions.

- Mental health service providers, including psychiatrists, clinical psychologists and social workers, should be available in every facility taking care of COVID-19 patients.

- Health workers treating patients during the COVID-19 pandemic should be aware of symptoms of depression, anxiety, insomnia and/or distress developing in their patients and colleagues.

\section{KEYWORDS}

Suicidality, COVID-19, distress, isolation, quarantine

\section{CASE DESCRIPTION}

A 34-year-old man with a medical history of diabetes mellitus and smoking, presented to the emergency room with a 4-day history of weakness, fever and cough. On admission, his temperature was $37.5^{\circ} \mathrm{C}$, pulse was $102 \mathrm{bpm}$, blood pressure was $133 / 85 \mathrm{mmHg}$, and oxygen saturation was $96 \%$ while breathing ambient air. Breath sounds were diminished with crackles over the left lower lung field. Chest radiographs showed interstitial opacities in the left lower lobe. Laboratory tests were unremarkable except for mild lymphopenia and thrombocytopenia. Nasopharyngeal and oropharyngeal swabs for COVID-19 were obtained due to a history of exposure to COVID-19 patients. After a positive result was reported, the patient was admitted to an isolation ward. Treatment with antibiotics and hydroxychloroquine was initiated. Direct contact between the staff and the patient was purposely minimal; interaction was primarily via an electronic audio-video link. Visitors were prohibited. During his hospitalization, the patient had no fever or respiratory distress. However, he developed symptoms consistent with anxiety and insomnia. 
A supportive intervention was initiated by a team of social workers and treatment with brotizolam was commenced.

On hospital day 7, the patient attempted suicide by jumping from the third-floor ward. Droplet and contact precautions were initiated, and he was transferred to the trauma bay. All staff caring for the patient wore personal protective equipment. The patient was intubated and transferred under strict infection control measures for computed tomography which revealed subdural and subarachnoid haemorrhages, bladder laceration and pelvic fracture with active bleeding. Due to haemodynamic instability, the patient was urgently transferred to the operating room for pelvic stabilization. The procedure was performed in a distal operating room and all possible precautions were taken to prevent infection spread and protect the staff, including shutting down air-conditioning and postponing other surgeries in the compound. After pelvic fixation, the patient was transferred to a level 1 trauma centre for neurosurgical intervention. Full sanitization of the operating room, trauma bay and radiology unit was carried out.

\section{DISCUSSION}

More than 1000000 people worldwide have been infected with COVID-19. Most of these patients are either hospitalized in isolated and specially designed clinical wards or self-quarantined at home. Although most of the global attention has been focused on the treatment of the respiratory and cardiovascular complications of the disease, a large proportion of COVID-19 patients are experiencing psychological problems, including anxiety, depression and stress ${ }^{[1]}$. Patients infected with a novel, potentially fatal virus may experience fear of the disease itself and also loneliness and anger, and anxiety for the welfare of friends and family. All of these emotions may be aggravated by fever, dyspnoea and weakness, as well as the adverse effects of treatments such as chloroquine and corticosteroid administration. Studies performed after the 2003 severe acute respiratory syndrome (SARS) and 2014 Ebola outbreaks indicated very high rates of psychological distress among those who had been quarantined. Suicide attempts were documented among some hospitalized patients. Long-term behavioural changes were also reported ${ }^{[2,3]}$.

Most of the healthcare workers treating COVID-19 patients, usually working in internal medicine wards and ICUs, have only basic training in providing mental health care. A large proportion of the workers themselves develop symptoms of depression, anxiety, insomnia and/or distress ${ }^{[4]}$. Mental health service providers, including psychiatrists, clinical psychologists and social workers, should be made available in every facility taking care of COVID-19 patients. These services should be readily available through safe communication channels. Regular screening for depression, anxiety and suicidality should be instituted for both patients and healthcare personnel. Special attention should be paid to those with pre-existing psychiatric disease. Early detection of stress reactions may enable simple, efficient intervention to prevent further morbidity and mortality, and lessen the burden on already overwhelmed healthcare systems ${ }^{[5]}$.

\section{CONCLUSION}

Based on our unfortunate experience, we encourage clinicians to pay special attention to the mental health aspects of the COVID-19 pandemic.

\section{REFERENCES}

1. Xiang YT, Yang Y, Li W, Zhang L, Zhang Q, Cheung T, et al. Timely mental health care for the 2019 novel coronavirus outbreak is urgently needed. Lancet Psychiatry 2020;7(3):228229.

2. Brooks SK, Webster RK, Smith LE, Woodland L, Wessely S, Greenberg N, et al. The psychological impact of quarantine and how to reduce it: rapid review of the evidence. Lancet 2020;395(10227):912-920.

3. Barbisch D, Koenig KL, Shih FY. Is there a case for quarantine? Perspectives from SARS to Ebola. Disaster Med Public Health Prep 2015;9(5):547-553.

4. Lai J, Ma S, Wang Y, Cai Z, Hu J, Wei N, et al. Factors associated with mental health outcomes among health care workers exposed to coronavirus disease 2019. JAMA Netw Open 2020;3(3):e203976.

5. Folkman S, Greer S. Promoting psychological well-being in the face of serious illness: when theory, research and practice inform each other. Psychooncology 2000;9(1):11-19. 